Sociologie et sociétés

\title{
La confrontation des féministes en particulier au racisme en
} général

\author{
Remarques sur les relations du féminisme à ses sociétés
}

\section{Colette Guillaumin}

Volume 49, numéro 1, printemps 2017

URI : https://id.erudit.org/iderudit/1042811ar

DOI : https://doi.org/10.7202/1042811ar

Aller au sommaire du numéro

Éditeur(s)

Les Presses de l’Université de Montréal

ISSN

0038-030X (imprimé)

1492-1375 (numérique)

Découvrir la revue

Citer cet article

Guillaumin, C. (2017). La confrontation des féministes en particulier au racisme en général : remarques sur les relations du féminisme à ses sociétés. Sociologie et sociétés, 49(1), 155-162. https://doi.org/10.7202/1042811ar 


\section{La confrontation des féministes en particulier au racisme en général}

Remarques sur les relations du féminisme

à ses sociétés'

\section{COLETTE GUILLAUMIN}

CNRS-URMIS

E féminisme, sous Ce nom et comme mouvement contestataire collectif,
est né en même temps — dans la première moitié du $19^{e}$ siècle - que le mouvement (contestataire collectif) anti-esclavagiste. Non seulement en même temps mais entretenant avec lui des liens fondateurs, ceci en deux sens: en ce que certains de ses membres l'étaient également du mouvement contre l'esclavage et en ce que l'un des moments fondateurs du féminisme contemporain, la rencontre de Seneca Falls, est en partie issue d'un conflit au sein du mouvement anti-esclavagiste. On peut d'ailleurs voir une photo prise lors d'un meeting de Frederick Douglass, ancien esclave, figure déterminante du mouvement anti-esclavagiste, où l'assistance est composée de plus de femmes que d'hommes. ${ }^{1}$

Le rôle joué par Le Deuxième Sexe (1949) dans la renaissance féministe des années soixante de notre siècle est évident, quelle que soit en cette occurrence la nature attribuée à ce texte: celle d'être un mythe de référence ou celle d'initiateur ou de catalyseur

1. Ce texte a été initialement publié en 1998 par l'Association Nationale des Études Féministes (ANEF). GUILLAUMIN, C. (1998), «La confrontation des féministes en particulier au racisme en général. Remarques sur les relations du féminisme à ses sociétés", paru dans «Les féministes face à l'antisémitisme et au racisme», Journée de l'ANEF du 14 Juin 1997, Supplément du Bulletin de l'Association Nationale des Études Féministes, $n^{\circ} 26$, p. 7-14. 
du mouvement. L'introduction que Simone de Beauvoir a faite à son livre place d'emblée, dans une perspective sociale et historique, les femmes dans une situation analogue (ce qui n'est pas dire semblable) à celle d'autres groupes infériorisés: «[...] qu'il s'agisse d'une race, d'une caste, d'une classe, d'un sexe réduits à une condition inférieure, les processus de justification sont les mêmes».

Les similitudes entre le féminisme et d'autres mouvements d'émancipation sont à la fois pratiques et théoriques. Elles relèvent d'une expérience commune, celle d'une situation comparable dans le système social et celle de la protestation contre cette situation; sur le plan théorique, elles impliquent une description parente des systèmes d'oppression. Mais plus, c'est une constante que, parmi les minoritaires, les femmes quelles qu'elles soient, comme les juifs en général, sont plus fréquemment engagées dans les mouvements d'émancipation et de libération d'autres groupes que le leur ou à visée universelle. Et que elles/ils le sont également, plus fréquemment, dans l'étude des phénomènes de minorité et de domination touchant des groupes autres que le leur. L'histoire des mouvements antiségrégationnistes aux États-Unis, anti-apartheid et anticolonialistes en Afrique et en Europe, comme le développement, dans les pays industrialisés, des recherches sur les discriminations et le racisme en témoignent. Quelle que soit la façon de le voir, il ne peut pas échapper à l'observation qu'il existe un lien entre les mouvements et préoccupations minoritaires et le féminisme, non seulement, bien sûr, parce qu'il est l'un d'entre eux mais aussi à travers les femmes qui sont leurs actrices, la conscience qu'elles ont de l'état de leur société et les projets qu'elles ont sur ce que devrait être cette société.

Quel caractère spécifique présente le mouvement ou la mouvance féministe si on les compare aux autres mouvements d'émancipation (ce terme est faible ou imprécis, mais je ne vois que lui pour recouvrir des projets aussi divers et parfois porteurs de contradictions que sont la liberté des individus et leur autonomie, les droits civiques, les aspirations nationales, les libérations coloniales, etc.) aux mouvements antiracistes ou aux mouvements de défense des droits humains (dits en France «Droits de l'Homme»), etc.?

D’abord, bien sûr sa spécificité est d'être concerné par les femmes, mais il n'est pas si évident de dire quelle est la nature de ce concernement. Proche des combats antidiscriminatoires et antiracistes, il est antisexiste par définition. L'antisexisme est le dénominateur commun de toutes ses formes. Mais ce dénominateur commun n'entraîne pas de facto un projet de société et surtout il n'implique nullement une analyse identique des formes que prend le sexisme, de leur mécanisme ou de leur cause. Et il est bien vrai que l'émancipation recherchée est toute différente selon qu'il y a ou non critique des formes sociales, selon qu'il y a projet de société ou bien absence de projet de société. Et pour qu'il y ait projet de société, encore faut-il d'abord analyser la société existante et être dans une position critique de cette société-là.

Or, il va sans dire, mais mieux vaut le rappeler, que la structure socio-sexuelle de notre société n'est pas majoritairement mise en cause elle-même, elle semble en quelque sorte normale (améliorable certainement, mais normale), à coup sûr, aux mouvements féminins (qui se proposent de défendre les femmes et les intérêts des 
femmes «en tant que femmes»), mais également à une partie du féminisme qui peut réserver ses critiques de l'organisation sociale aux autres sociétés, réputées porteuses d'un sexisme organique qui n'existerait pas (ou plus) ici. Et ceci précisément (qui n'est pas caricatural, même s'il se présente peu sous une forme aussi extrême ou explicite) est l'une des formes du racisme réel ou potentiel. C'est en tous cas l'une des raisons de l'irritation de féministes d'autres sociétés envers certaines féministes des sociétés industrielles. Et c'est l'un des effets du refus d'analyser ce que sont les hommes et les femmes comme la relation qui les fait tels dans les sociétés que nous connaissons.

Avec une ironie toute relative, on pourrait sans doute aborder la diversité des mouvements de femmes à travers leur mode d'intervention sociale selon qu'ils seraient dans une optique «corporatiste», «syndicale» ou "politique». Et cela a quelque rapport avec la possibilité de poser la question du racisme dans les pratiques et dans les formations mentales. En fait, c'est la question de la définition elle-même du féminisme qui est sous-jacente, ce qui n'est pas une question académique, ni d'une façon générale, ni en ce qui nous préoccupe. On pourrait penser que le féminisme étant un mouvement antisexiste et non pas un mouvement antiraciste ou anti-impérialiste, la question du racisme et de l'antisémitisme se poserait en fonction d'événements déterminés et qu'elle interviendrait ponctuellement à des moments précis, bref qu'elle tiendrait à des occurrences extérieures.

Ce pourrait parfaitement être le cas dans une perspective qui ne voudrait - qui ne veut - considérer que les intérêts des «femmes en tant que femmes", c'est-à-dire explicitement et intrinsèquement en tant que piliers de leur communauté, définies par les hommes de cette communauté à laquelle et auxquels elles appartiennent. C'est une forme corporatiste en quelque sorte de défense et promotion des intérêts d'un groupe professionnel, celui des épouses et mères, lequel étant recruté parmi les êtres humains femelles en conclut que tous les êtres humains femelles sont obligatoirement épouses et mères et seulement cela. Ce dont les hommes tombent d'accord si on le leur demande. Ce corporatisme serait la défense des vraies femmes, lesquelles sont des défenseures inconditionnelles (par définition) de leurs hommes dans le champ politique des antagonismes nationaux, communautaires, de classe, etc. Elles n'ont a priori pas de raison particulière, si ce n'est celle de la pitié humaine, de considérer qui que ce soit d'autre comme digne d'intérêt, de droits, de liberté, et parfois de vie. Ce n'est pas une forme rarissime de mouvement féminin et qui se conçoit bien, en effet, comme un mouvement de défense des intérêts des femmes. Les associations de femmes des systèmes nationalistes et communautaristes comme des systèmes totalitaires ou religieux, répondent en fait à cette conception des femmes comme éléments d'une communauté où elles doivent prendre leur place, toute leur place et seulement leur place. Le système politique de la communauté où elles sont nées est le leur, raciste ou antiraciste, c'est selon (mais le plus souvent raciste).

La configuration est différente si on envisage les intérêts des femmes dans une perspective «syndicale» qui pourrait se voir comme la défense des femmes certes, mais également l'acquisition de droits meilleurs ou plus équitables, bref une conquête et une 
recomposition de la distribution sociale, celle des rôles et celle des biens, de façon à ce que hommes et femmes atteignent une sorte d'équilibre statutaire de partenaires, sans d'ailleurs que le statut de «femme» et celui d' «homme» soient eux-mêmes interrogés. La perspective differe encore si on envisage le féminisme comme mouvement "politique», c'est-à-dire comme un mouvement qui a un projet de société ou qui cherche à en produire un, dont la réflexion est orientée par force dans ce sens. Par force, dans la mesure où l'analyse et la critique de la structure socio-sexuelle ne peuvent pas ne pas remettre en cause l'ensemble de l'organisation sociale. Ce qui fait du lesbianisme dans le féminisme (je ne dis pas l'homosexualité, qui est autre chose) une position forcément politique, qui oblige à penser autrement que par le biais du sexe. Le lesbianisme ne peut être ni corporatiste (être lesbienne n'est pas un métier) ni syndical (lesbienne n'est pas un statut de sexe). Ancienne question certes, mais nullement vieille dans le mouvement féministe.

On a aperçu que la «défense du droit des femmes» et l'antisexisme ne sont pas nécessairement liés à une préoccupation d'émancipation, parfois même au contraire et que par conséquent une partie des «mouvements féminins» se place hors le projet d'émancipation. Dans son principe, un mouvement d'émancipation, s'affrontant aux formes de la domination, mais aussi de la contrainte et de l'exploitation, me semble-til, ne peut pas ne pas avoir une vision au moins, sinon un projet d'ensemble d'une société possible vers laquelle tendre et ne peut pas éviter ces questions sur le fond. Et s'il ne les pose pas, s'il ne se les pose pas, il pourra, à terme et parfois dans l'immédiat, entreprendre des actions qui travailleront contre les femmes. Et en prônant la différence (par exemple), revenir là même d'où il tentait de sortir. Qu'est-ce, en effet, que «les intérêts des femmes»? Parfois, on croit répondre en demandant: «les intérêts de quelles femmes?», mais c'est une mauvaise question. Ce ne sont pas les femmes qui sont différentes (quoique bien évidemment elles le soient dans leur existence quotidienne), ce sont leurs choix politiques qui le sont. Et ensuite, ce sont leurs possibilités matérielles qui le sont et ne permettent pas les mêmes décisions pratiques. Probablement, il s'agit là de l'un des conflits majeurs au sein des mouvements de femmes, si ce n'est le conflit majeur. C'est également dans ce clivage politique profond que la possibilité de poser - ou non - la question d'une organisation sociale qui ne soit pas raciste réside. Celle où nous vivons l'est. Où vivent les féministes, objets du racisme et/ou productrices de racisme.

Les différentes formes de mouvements féminins et de féminisme sont engagées et partie prenante de l'histoire politique de leurs sociétés. Ce que nous montre d'ailleurs, en ce qui concerne le féminisme ( «syndical» et "politique», en résumé le féminisme de l'émancipation justement), les attaques qu'il rencontre. Telle, par exemple, la levée de boucliers des «analystes» sociologiques ou politiques de la situation algérienne, contre les féministes explicites, distinguées des femmes qui seraient, elles, des vraies femmes et dont le courage «modeste» légitimerait une lutte «raisonnable». Ce que montre également la capacité étonnante de déni de l'action des féministes, déni si évident dans la proposition que ce qu'elles obtiennent serait arrivé de toute façon, car 
conforme à «l'évolution de la société». Les féministes sont des citoyens que la structure sociale, l'organisation et le fonctionnement de leur société concernent qu'elles le veuillent ou non, puisque, en effet, elles interviennent dans cette organisation. Dont aujourd'hui le racisme, dont l'antisémitisme est l'une des formes, est un trait structurel.

En fait, une bonne part des analyses du racisme repose sur, ou implique, le présupposé qu'il serait un phénomène autonome, sorte d'excroissance ou de «corps étranger» dans la société où il se produit. Cependant l'antisémitisme et le racisme nazi, l'apartheid, la ligne de couleur aux États-Unis, en France l'antisémitisme, de l'affaire Dreyfus aux lois antisémites de l'État français (1940-1944) et à sa continuité dans le champ politique avec le Front National, le racisme contre les Maghrébins et les Africains, ne sont pas des phénomènes «extérieurs» à leur société, ils lui sont intrinsèquement liés. Ce ne sont pas d'incompréhensibles accidents. Ils sont inscrits dans les lois ou «institutionnels», ce qu'on nomme systémique dans les sciences sociales, ce qui suppose un processus de mise en œuvre d'une intention politique délibérée. Mais plus, dans certaines formes sociales, à certains moments historiques, le racisme est le fondement du projet social. Comme la soumission des femmes et leur appropriation, qui sont factuellement le socle des sociétés historiquement et actuellement connues, sont explicitement un constituant fondamental des formes nationalistes, communautaires (là encore, ces termes sont imprécis mais acceptons-les provisoirement pour désigner les projets de fermeture sur soi et d'exclusion comme d'exaltation du groupe).

Sans analyse des formes sociales, on traite racisme et sexisme comme des épiphénomènes ou des affaires conjoncturelles, bref des sortes de dysfonctionnement. On s'empêche ainsi, on s'interdit même, de voir comment les ségrégations, les inégalités matérielles, la dépendance, de situations de fait se transforment en pratiques institutionnelles, en règles et structures sociales, en lois. Et de factuelles deviennent intentionnelles et organiques. Une illusion à laquelle il est tentant de succomber voudrait qu'un mouvement d'émancipation, un mouvement minoritaire né de la persécution, de la connaissance de l'oppression ou de la contrainte, devrait les reconnaître en toutes circonstances et dans tous les groupes qui en sont les cibles. Et surtout ne jamais les pratiquer, ni c'est le moins - les relayer. Illusion, en effet. Et singulière expérience que de voir, parmi les siennes, au plus proche de soi, le déni parfois, l'ignorance souvent. Et à certains moments, brutalement, le relais et l'adoption du racisme de la société banale.

L'arrogance raciste a une série de conséquences, dont d'aveugler les femmes visà-vis les unes des autres. Cette arrogance est l'expression d'un rapport de force où certains groupes sont à la merci d'autres groupes. Et les femmes appartiennent à ces groupes, à tous et à chacun de ces groupes. Et quand on dit ici "appartiennent», c'est aux deux sens du terme qu'il faut l'entendre: au sens propre et matériel d'appartenir aux hommes de ce groupe (qui décident de la forme et des actions du groupe). Et au sens figuré, qui est tout autant fondateur, d'appartenir à cette histoire, à cette culture, à cette langue, à cette classe, à cette religion, etc., bref au sens de manière d'être au monde et au sens de conscience. Or l'arrogance raciste s'exprime précisément dans le déni du rapport de force lui- même et dans le déni des effets du rapport de force. Au 
plus, elles les prétend imaginaires ou secondaires, attribuant alors une tournure d'esprit «victimiste» à celles qui en sont l'objet. A ce propos, le reproche de «victimisation », si souvent repris, dans une perspective de disqualification, à l'encontre du féminisme et plus souvent encore à l'intérieur du mouvement, est une constante du discours raciste lui-même. Il intervient dans les circonstances politiques où un groupe discriminé dit qu'il l'est, dit comment il l'est et dit que ce n'est pas admissible. On appelle cela «se poser en victimes». Comme s'ils décidaient, eux, d'être victimes, comme s'il s'agissait dans leurs propos de pure incantation, sans fondement réel alors même qu'ils parlent des moyens employés contre eux pour les maintenir dans la sujétion, la dépendance ou la fragilité, des moyens employés contre eux pour les tuer. C’est une opération de disqualification sans doute, mais c'est aussi une opération de déni. C'est dénier d'abord la sujétion et la dépendance mais également les pratiques qui les accompagnent, les bénéfices qui en sont tirés, les conséquences de cet assujettissement. C'est dans le racisme d'abord que j'ai remarqué ce biais particulier du déni qu'est l'accusation de victimisation et sans doute cela m'a-t-il aidé à comprendre ce qui se passait à l'encontre du féminisme (et des femmes) et dans le mouvement lui-même. Arrogance raciste, dont l'envers est une culpabilité affichée (un sentiment de culpabilité) tout théorique et formel, forme d'hommage du vice à la vertu et qui dispense tout autant de considérer les faits, qui ne fait que revêtir l'aveuglement du manteau de la respectabilité.

À quoi est-on aveugle ici? Aux rapports de force impliqués. Doubles et toujours à l'œuvre ensemble.

- Ceux qui soumettent certains groupes aux autres ou soumettent les autres à eux (car ce n'est pas une situation symétrique, l'un de ses caractères spécifiques étant, justement, la dissymétrie). Quand on parle de groupe ici, on désigne les ensembles sociaux maintenus par la reproduction, quel que soit par ailleurs leur caractère particulier: classe, religion, nation, culture, «race», etc.

- Ceux qui mettent les femmes à la merci des hommes, de leur groupe précisément, lesquels sont constitutifs des précédents et leur sont organiquement liés au sens strict du terme.

Ce sont à ces derniers rapports que se confrontent les féministes. Explicitement. Et elles sont obligatoirement confrontées aux premiers dans la mesure où les relations des femmes aux hommes sont partie prenante des relations des hommes entre eux. Penser aux femmes, penser les femmes, oblige à prendre en compte les deux termes. Appartenir à certains groupes permet ou bien empêche d'être lesbienne (je ne dis pas homosexuelle). Appartenir à certains groupes confronte directement aux hommes auxquels on appartient, mais pas à tous les hommes. Appartenir à certains groupes signifie être tué pour être né dans ce groupe et tué avec le groupe dans son ensemble. Appartenir à certains groupes signifie être ségrégué ou emprisonné ou chassé ou discriminé pour appartenir à ce groupe, avec le groupe dans son ensemble. Appartenir à certains groupes confronte directement aux hommes auxquels on appartient et confronte, de 
surcroît et souvent d'abord, aux hommes qui tiennent à merci les hommes auxquels on appartient. Appartenir à certains groupes vous met dans la position d'enjeu, de proie ou de moyen dans la guerre que mènent ces groupes avec d'autres ou dans la guerre qu'ils sont forcés de subir (etc., hélas!). Car, en effet, dans la mesure même où les femmes sont sociologiquement fonction des hommes (dans la dépendance et «à la merci » est la forme que prend cette fonction de type algébrique), les féministes, comme les femmes, sont diverses. Et ce qui les met ensemble est en même temps très simple - leur commune domination par les hommes et très complexe — les hommes des différents groupes ne sont pas dans une relation neutre et symétrique où chaque groupe n'existerait que par des caractéristiques aléatoires, idéalement équivalentes. Et les femmes ne flottent pas au-dessus de la mêlée, mais sont bien de leur histoire, de leur langue, de leur culture et plus, de la place que leur assigne leur groupe de naissance dans l'ensemble des relations pas du tout neutres et égalitaires qu'entretiennent entre eux ces divers groupes.

La tentative d'en sortir avec cette fameuse «différence» des femmes qui les ferait toutes semblables en face des hommes, toutes semblables par nature, métaphysiquement, revient précisément à éviter de connaître et d'affronter le rapport de force: en affirmant une spécificité d'essence féminine, on voile sûrement les rapports de force avec les hommes, mais on dénie également les rapports de force entre les hommes, dont les femmes sont partie prenante malgré elles (mais aussi parfois très volontairement). Ou bien, en faisant appel à la spécificité des appartenances, nommée diversité culturelle, où toutes les femmes sont différentes les unes des autres selon l'histoire et la place de leur groupe d'appartenance et ainsi résorbées dans leur groupe, déniées. Dans les deux cas, la renonciation à la compréhension de ce qui fait les femmes est complète. De ce qui construit les femmes et les hommes (qui n'existent pas l'un sans l'autre, cela va sans dire, mais tant et si bien qu'on finit par n'y plus penser). De la relation de sexe dans les groupes sociaux de reproduction. De l'usage et de l'instrumentalisation des femmes, de leur assignation et de leur place d'objet et d'outil de la transmission et de la reproduction. Ce contre quoi une part non négligeable d'entre elles se révolte et ce de différentes façons selon les possi- bilités et les circonstances. (Mais toutes ne s'insurgent pas.) Cette révolte n'est pas dirigée contre ce qui, du groupe, constitue l'individu, ce qui les fait elles- mêmes, nous fait nous-mêmes : langue, histoire, culture, dont nous sommes; mais contre notre instrumentalisation et notre usage par les hommes. Il est tout à fait erroné, il me semble, de dire «en tant que femme ET en tant que $\mathrm{x}, \mathrm{z}$ ou $n »$. Car ce sont les relations de pouvoir entre groupes qui imposent l'idée d'une telle coupure. Un être humain est un, conscience et sujet pour lui-même. Il peut avoir à faire face à des situations conflictuelles ou complexes, mais c'est autre chose qu'une conscience multiple: lui-même est un sujet unique. L'idée de «la différence», celle d'une spécificité métaphysique du «féminin» ou bien celle de femmes "différentes» par immersion irréductible dans leur seule appartenance de groupe sont l'une et l'autre l'effet d'un affrontement refusé à la structure sexuelle de nos sociétés, du renoncement à la réflexion sur elle. Refus et renoncement dont les effets nous déchirent. 
Pour terminer, mais certainement pas pour finir (ou le contraire, je ne sais), quelques remarques de vocabulaire puisque les mots sont nos outils de travail. J'ai les plus grandes réserves, qui ne font que croître, sur les termes «patriarcat» et «genre». Je leur préfère «domination des hommes» et «sexe». En ce sens que «patriarcat» désigne un mode particulier, une variante, historiquement et géographiquement délimitée, de la domination des hommes et que «genre» finit par masquer plus ou moins le fait que le sexe anatomique qui est le déterminant social du genre (ce que signifie bien le terme) l'est obligatoirement et impérativement. Ce n'est pas central à notre préoccupation ici, encore que la dénomination soit dans l'analyse des rapports de sexe un point crucial. Cette analyse de ce que sont nos sociétés et des liens qu'entretient le racisme avec la division sexuelle, ses effets sur les femmes des différents groupes et leurs relations entre elles est, elle, indispensable. 